\title{
Revisit prompt $J / \psi$ production in associated with Higgs Boson via gluon fusion at the LHC
}

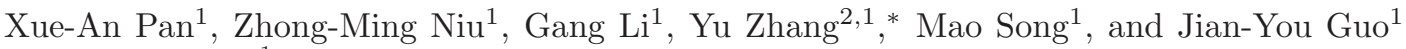 \\ 1 School of Physics and Materials Science, Anhui University, \\ Hefei, Anhui 230039, People's Republic of China and \\ 2 Institutes of Physical Science and Information Technology, \\ Anhui University, Hefei, Anhui 230039, People's Republic of China
}

(Dated: September 7, 2021)

\begin{abstract}
The production of charmonium associated with Higgs boson via gluon fusion has been investigated in Ref. 1], in which they considered the contribution of final Higgs boson radiation off the charm quark at tree level and found that this process is to be far too rare to be observable in any of the considered experiments. In this paper, the production of prompt $J / \psi$ associated with Higgs boson via gluon fusion at the $14 \mathrm{TeV}$ LHC within the factorization formalism of NRQCD is revisited. After considering the contribution from the final Higgs boson radiation off the top quark in the loop, which is more than three orders of magnitudes over the charm quark at tree level, the production of prompt $J / \psi$ associated with Higgs boson has great potential to be detected. The prompt $J / \psi$ production includes the direct production and indirect production via radiative or hadronic decays of high excited charmonium states. For the direct $J / \psi+H$ production via gluon fusion loop-induced, the ${ }^{3} S_{1}^{(8)}$ Fock state gives dominant contribution to the cross section, which is about $95 \%$ to the total direct production. The indirect contribution via loop-induced is appreciable, since the summation of which from $\psi(2 S)+H, \chi_{c 1}+H$ and $\chi_{c 2}+H$ is about $34 \%$ to the total cross section of prompt $J / \psi+H$. While the indirect contribution from $\chi_{c 0}+H$ is tiny, which can be neglected. With the great potential to be detected, prompt $J / \psi$ production in associated with Higgs boson can help us to further understand the mechanism of colour-octet, as well as can be useful to further investigate the coupling of the Higgs boson and fermion.
\end{abstract}

PACS numbers: 12.38.Bx, 13.85.Fb, 14.40.Lb, 14.80.Cp

\section{INTRODUCTION}

Since the discovery of the first heavy charmonium $J / \psi$ in 1974 2, 3], the production of heavy quarkonium has been an important investigating topic in hadron physics. An increasing number of experiments have studied the production process of heavy quarkonium in detail. For example, the inclusive charmonium production has been measured by BaBar [4] and Belle [5], the photoproduction of $J / \psi$ has been investigated by H1 [6 10] and ZEUS [11], and the hadronic and polarization productions of heavy quarkonium have been studied at hadron colliders 12 16. In addition, production processes of the double charmonium have been detected, such as $J / \psi+J / \psi[17-20], J / \psi+\chi_{c 0}$ 21] and $J / \psi+\eta_{c} 21,22$. In order to explain the production and decay of the heavy quarkonium, the colour-evaporation model (CEM) 23, 24], colour-singlet model (CSM) 25, 26] and nonrelativistic quantum chromodynamics (NRQCD) [27] have been proposed. At present, NRQCD is more widely accepted and applied to the production and decay of heavy quarkonium. In NRQCD factorization, the production and decay of the heavy quarkonium are factorized into the short-distance coefficients (SDCs) and long distance matrix elements (LDMEs). The SDCs can be perturbatively calculated by using the expansion of the strong-coupling constant $\alpha_{s}$, and the LDMEs are process-independent and universal, governed by nonperturbative QCD dynamics, and can be extracted from experiments. NRQCD has achieved remarkable success in explaining the puzzle of $J / \psi$ and $\psi(2 S)$ surplus production at the Tevatron[28, 29], subsequently it was used extensively to explain the production and decay of heavy quarkonium, and attained many phenomenological successes (see reviews [30 32] for details). However, there still exist some problems in NRQCD, such as it cann't provide a full description for the data of the double $J / \psi$ measured by the CMS [19, 33, 34], and the universality of LDMEs [35, 36] etc., which all indicate that the NRQCD needs further investigating.

Due to the clean signature, the associated productions of heavy quarkonium and standard model (SM) bosons are ideal channels to test the NRQCD, which have been attracted more and more attention. The hadronic productions of heavy quarkonium associated with $\gamma, W^{ \pm}, Z$ and Higgs boson have been studied at leading order (LO) within NRQCD [1, 37 39], and the next-to-leading order (NLO) QCD corrections for the productions of heavy quarkonium

*Electronic address: dayu@ahu.edu.cn 
associated with $\gamma, W^{ \pm}, Z$ have been calculated in Refs. 40 44. Furthermore, for the processes of $J / \psi+W^{ \pm}$and $J / \psi+Z$, the contributions from Single Parton Scattering (SPS) and Double Parton Scattering (DPS) have been investigated [45, 46]. In experimental, the productions of $J / \psi+Z, J / \psi+W^{ \pm}$at hadron colliders were also detected [47 49]. The production rate of $J / \psi+W^{ \pm}$measured by ATLAS [47] is an order of magnitude larger than SPS predictions from NRQCD [41, 50], and the gap can be filled by considering the contribution from DPS 46]. As for $J / \psi+Z$, even if taking the contribution from NLO CEM SPS + DPS [45] into account, the behavior of the transverse momentum distribution measured by ATLAS [48] exhibits slight different with the theoretical prediction. As regards the production of heavy quarkonium associated with Higgs boson, there is no positive news from both phenomenology and experiment.

In Ref. [1], Kniehl et al. estimated the production of the charmonium associated with Higgs boson at LO based on NRQCD at $e^{+} e^{-}, e^{ \pm} p, p \bar{p}$ and $p p$ colliders for the first time. They considered the contribution from the partonic processes $g+g \rightarrow \mathcal{Q}+H$ and $q+\bar{q} \rightarrow \mathcal{Q}+H$, where the $\mathcal{Q}$ is charmonium, $q$ is the light quark, and $H$ is the Higgs boson, and found that the process of heavy quarkonium associated with Higgs boson is to be far too rare to be observable in any of the considered experiments. We repeat the calculation in Ref. [1] for the direct production of $J / \psi+H$ and $\chi_{c J}+H$, and obtain consistent results of the transverse momentum and rapidity distribution. Adopting the same input parameters, we obtain the total cross section of $J / \psi$ production in associated with Higgs boson via gluon fusion at the $14 \mathrm{TeV} \mathrm{LHC}$ is about $0.02 \mathrm{fb}$, which is agree with the results shown in their another work [51]. One can see that, in this case it is difficult to detect this process at the LHC. We consider the contribution from the final Higgs boson radiation off top quark via loop-induced, and find that the production of heavy charmonium associated with Higgs boson can be potentially detected at the LHC $^{1}$.

In this work, we will revisit the production of prompt $J / \psi$ associated with Higgs boson via gluon fusion at the LHC within the NRQCD factorization formalism. The final Higgs boson can produce from two sources, including radiation off the charm quark (lablled as S1 in the following) and off the top quark via loop-induced (lablled as S2). Noted that S1 has been considered by Kniehl et al. in Ref.[1], and S2 will be considered for the first time in this work. Since the production rate from the partonic process $q+\bar{q} \rightarrow \mathcal{Q}+H$ is tiny, we will not consider it. Besides the direct production, prompt $J / \psi$ candidates that can also be produced indirectly via radiative or hadronic decays of heavier charmonium states, such as $\chi_{c J} \rightarrow J / \psi+\gamma(J=0,1,2)$ and $\psi(2 S) \rightarrow J / \psi+X$. We find that the contribution of S2 is more than three orders of magnitudes larger than $\mathrm{S} 1$, and the ${ }^{3} S_{1}^{(8)}+H$ production channel plays an important role in $J / \psi+H$ production via $g g$ fusion loop-induced. With the detectable potential at the LHC, this process can be useful to test the heavy quarkonium production mechanism and investigate the coupling of Higgs and fermion.

\section{THE DETAILS OF THE CALCULATION FRAMEWORK}

In this section, we introduce the detail of the calculation for prompt $J / \psi$ production in associated with $H$ via gluon fusion, $p p \rightarrow g g \rightarrow J / \psi+H$, at the LHC in NRQCD. The total cross section of prompt $J / \psi+H$ production can be expressed as follow

$$
\begin{aligned}
\sigma^{\text {prompt }}(J / \psi+H)= & \sigma^{\text {direct }}(J / \psi+H)+\sigma^{\text {indirect }}(\text { From } \psi(2 S)+H)+\sigma^{\text {indirect }}\left(\text { From } \chi_{c J}+H\right) \\
= & \sigma(p p \rightarrow g g \rightarrow J / \psi+H)+\sigma(p p \rightarrow g g \rightarrow \psi(2 S)+H) \times \operatorname{Br}(\psi(2 S) \rightarrow J / \psi+X)+ \\
& \sum_{J=0}^{2} \sigma\left(p p \rightarrow g g \rightarrow \chi_{c J}+H\right) \times \operatorname{Br}\left(\chi_{c J} \rightarrow J / \psi+\gamma\right),
\end{aligned}
$$

where the $\sigma^{\operatorname{direct}}(J / \psi+H)$ is the cross section of the direct contribution from $J / \psi+H$ production; $\sigma^{\text {indirect }}\left(\right.$ From $\left.\psi(2 S) / \chi_{c J}+H\right)$ denotes the indirect contribution due to hadronic/radiative decays of $\psi(2 S) / \chi_{c J}$ from $\psi(2 S) / \chi_{c J}+H$ production. $\operatorname{Br}(\psi(2 S) \rightarrow J / \psi+X)$ and $\operatorname{Br}\left(\chi_{c J} \rightarrow J / \psi+\gamma\right)$ respectively represent the branching ratio of $\psi(2 S)$ and $\chi_{c J}$ decaying into $J / \psi$, which can be obtained from [52] as

$$
\begin{aligned}
\operatorname{Br}(\psi(2 S) \rightarrow J / \psi+X) & =(61.4 \pm 0.6) \%, \\
\operatorname{Br}\left(\chi_{c 0} \rightarrow J / \psi+\gamma\right) & =(1.40 \pm 0.05) \%, \\
\operatorname{Br}\left(\chi_{c 1} \rightarrow J / \psi+\gamma\right) & =(34.3 \pm 1.0) \%, \\
\operatorname{Br}\left(\chi_{c 2} \rightarrow J / \psi+\gamma\right) & =(19.0 \pm 0.5) \% .
\end{aligned}
$$

\footnotetext{
${ }^{1}$ Note that final Higgs boson can also radiate off bottom quark via loop-induced. The contribution of the bottom-loop is about one order of magnitude large than that considered in Ref.[1], but nearly three orders of magnitude smaller than that of top-loop. Therefore, we don't consider it in this work.
} 
$\sigma(p p \rightarrow g g \rightarrow \mathcal{Q}+H)$ denotes the cross section for the production of charmonium $\mathcal{Q}$ associated with $H$ via gluon fusion, which can be factorized as

$$
\begin{aligned}
\sigma(p p \rightarrow g g \rightarrow \mathcal{Q}+H)= & \int d x_{1} d x_{2} f_{g / A}\left(x_{1}, \mu_{f}\right) f_{g / B}\left(x_{2}, \mu_{f}\right) \\
& \times \sum_{n}\left\langle\mathcal{O}^{\mathcal{Q}}[n]\right\rangle \hat{\sigma}(g+g \rightarrow c \bar{c}[n]+H)
\end{aligned}
$$

Here $f_{g / A, B}\left(x_{1,2}, \mu_{f}\right)$ is the distribution function of the gluon radiated by proton $A$ or $B ; x_{1 / 2}$ denotes the momentum fraction of the proton $A / B$ momentum carried by the gluon; the summation is taken over the possible intermediate Fock states $n={ }^{2 S+1} L_{J}^{(1,8)}$ of heavy $c \bar{c}$ pair; $\left\langle\mathcal{O}^{\mathcal{Q}}[n]\right\rangle$ is the LDME, which describes the process for the Fock state $c \bar{c}[n]$ evolving into an observable physical state $\mathcal{Q}$ by radiating soft gluons, with $\mathcal{Q}=J / \psi, \psi(2 S), \chi_{c J}(J=0,1,2)$ in our calculation. The relations between the LDMEs of various Fock states are

$$
\begin{aligned}
\left\langle O^{J / \psi, \psi(2 S)}\left[{ }^{3} P_{J}^{(8)}\right]\right\rangle & =(2 J+1)\left\langle O^{J / \psi, \psi(2 S)}\left[{ }^{3} P_{0}^{(8)}\right]\right\rangle, \\
\left\langle O^{\chi c J}\left[{ }^{3} S_{1}^{(8)}\right]\right\rangle & =(2 J+1)\left\langle O^{\chi_{c 0}}\left[{ }^{3} S_{1}^{(8)}\right]\right\rangle, \\
\left\langle O^{\chi_{c J}}\left[{ }^{3} P_{J}^{(1)}\right]\right\rangle & =(2 J+1)\left\langle O^{\chi_{c 0}}\left[{ }^{3} P_{0}^{(1)}\right]\right\rangle .
\end{aligned}
$$

$\hat{\sigma}(g+g \rightarrow c \bar{c}[n]+H)$ describes the short-distance cross section for the partonic process $g\left(p_{1}\right)+g\left(p_{2}\right) \rightarrow c \bar{c}[n]\left(p_{3}\right)+$ $H\left(p_{4}\right)$ via gluon fusion, which can be expressed as

$$
\hat{\sigma}(g+g \rightarrow c \bar{c}[n]+H)=\frac{1}{16 \pi \hat{s} N_{c o l} N_{p o l}} \int_{\hat{t}_{\min }}^{\hat{t}_{\max }} d \hat{\bar{t}}\left|A_{S, L}\right|^{2}
$$

Here $\hat{s}$ and $\hat{t}$ are Mandelstam variables, which are defined as $\hat{s}=\left(p_{1}+p_{2}\right)^{2}$ and $\hat{t}=\left(p_{1}-p_{3}\right)^{2}$, respectively. $N_{\text {col }}$ $\left(N_{\text {pol }}\right)$ refers to the colour (polarization) quantum number of Fock states $c \bar{c}[n]$. The summation is taken over the initial state and the final state, and the bar means averaging the colour and spin of the initial parton. $A_{S, L}$ can be obtained by the covariant projection method in Ref.[53]. The polarization summations of the Fock state $c \bar{c}[n]$ are

$$
\begin{aligned}
\sum_{J_{z}} \varepsilon_{\alpha} \varepsilon_{\alpha^{\prime}}^{*} & =\Pi_{\alpha \alpha^{\prime}} \\
\sum_{J_{z}} \varepsilon_{\alpha \beta}^{0} \varepsilon_{\alpha^{\prime} \beta^{\prime}}^{0 *} & =\frac{1}{3} \Pi_{\alpha \beta} \Pi_{\alpha^{\prime} \beta^{\prime}}, \\
\sum_{J_{z}} \varepsilon_{\alpha \beta}^{1} \varepsilon_{\alpha^{\prime} \beta^{\prime}}^{1 *} & =\frac{1}{2}\left(\Pi_{\alpha \alpha^{\prime}} \Pi_{\beta \beta^{\prime}}-\Pi_{\alpha \beta^{\prime}} \Pi_{\alpha^{\prime} \beta}\right) \\
\sum_{J_{z}} \varepsilon_{\alpha \beta}^{2} \varepsilon_{\alpha^{\prime} \beta^{\prime}}^{2 *} & =\frac{1}{2}\left(\Pi_{\alpha \alpha^{\prime}} \Pi_{\beta \beta^{\prime}}+\Pi_{\alpha \beta^{\prime}} \Pi_{\alpha^{\prime} \beta}\right)-\frac{1}{3} \Pi_{\alpha \beta} \Pi_{\alpha^{\prime} \beta^{\prime}}
\end{aligned}
$$

with

$$
\Pi_{\alpha \beta}=-g_{\alpha \beta}+\frac{p_{3 \alpha} p_{3 \beta}}{M_{Q}}
$$

Here $p_{3}\left(M_{Q}\right)$ is the momentum (mass) of quarkonium $\mathcal{Q}$.

The polarization summation of the two initial gluons for $g+g \rightarrow c \bar{c}[n]+H$ process is expressed as follows

$$
-g_{\mu \nu}+\frac{p_{\mu} \eta_{\nu}+p_{\nu} \eta_{\mu}}{p \cdot \eta}
$$

where $p$ is the momentum of initial gluon, and $\eta$ is an arbitrary light-like vector with $p \cdot \eta \neq 0$. In our calculation, $\eta$ is taken as the momentum of another initial gluon.

The Feynman diagrams for the process of $g+g \rightarrow c \bar{c}[n]+H$ from the contribution of S1 and S2 are generated with FeynArts [54], which are presented in Fig. 1 and Fig. 2 respectively, and we further reduce the Feynman amplitudes using FeynCalc [55], Apart [56, 57] and FIRE[58, 59]. Finally, we use LoopTools and FormCalc [60] to implement the numerical calculations. 


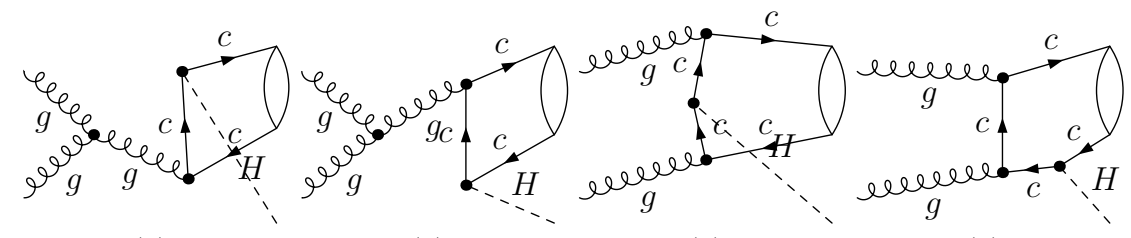

(1)

(2)

(3)

(4)

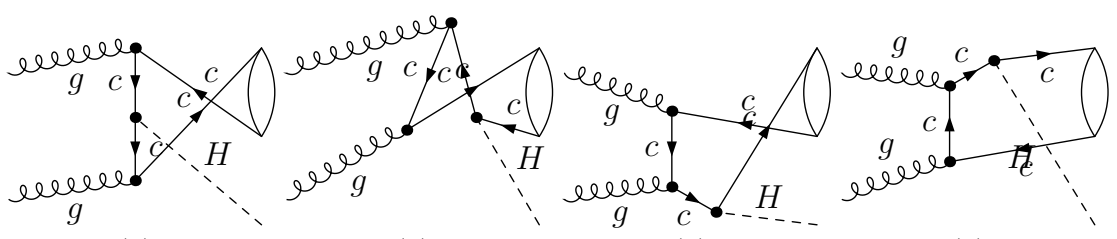

(5)

(6)

(7)

(8)

FIG. 1: Feynman diagrams for the partonic process $g+g \rightarrow c \bar{c}[n]+H$ via $g g$ fusion with final Higgs boson radiation off charm quark.

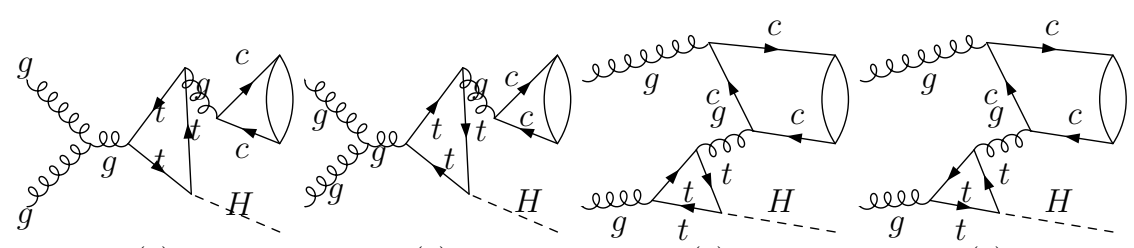

(1)

$(2)$

(3)

(4)

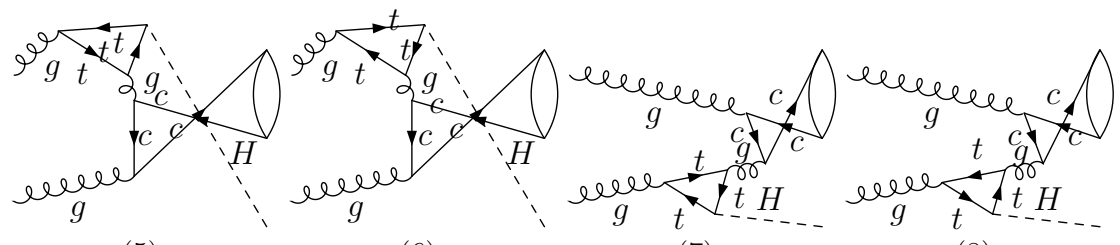

(5)

(6)

(7)

(8)

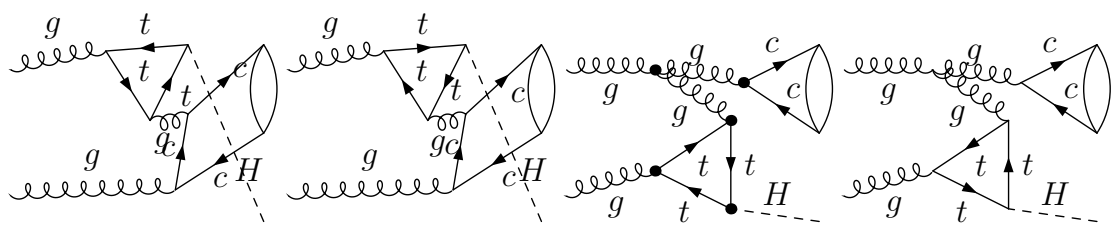

(9)

(10)

(11)

(12)
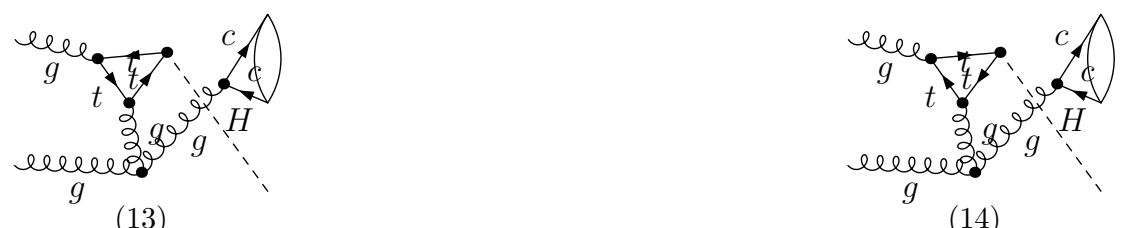

(14)

FIG. 2: Feynman diagrams for the partonic process $g+g \rightarrow c \bar{c}[n]+H$ via $g g$ fusion with final Higgs boson radiation off top quark in the loop.

\section{NUMERICAL RESULTS}

In what follows, we present numerical results for the production of prompt $J / \psi$ associated with Higgs boson via $g g$ fusion from the contribution of S1 and $\mathrm{S} 2$ at the LHC with $\sqrt{s}=14 \mathrm{TeV}$, respectively. The masses of the charm quark, top quark and Higgs boson are taken as $m_{c}=1.5 \mathrm{GeV}, m_{t}=172 \mathrm{GeV}$, and $m_{H}=125 \mathrm{GeV}$. The mass of the charmonium $\mathcal{Q}$ is taken as $M_{\mathcal{Q}}=2 m_{c}$, and we set cut $p_{T}^{\mathcal{Q}}>3 \mathrm{GeV}$ for final charmonium $\mathcal{Q}$, where the $p_{T}^{\mathcal{Q}}$ is the transverse momentum of charmonium $\mathcal{Q}$. In our calculation, the factorization scale and PDFs are chosen as $\mu_{f}=\sqrt{\left(p_{T}^{\mathcal{Q}}\right)^{2}+M_{\mathcal{Q}}^{2}}$ and CTEQ6L1 61], respectively. 
The LDMEs of $J / \psi$ is chosen from 62, 63] as

$$
\begin{aligned}
\left\langle\mathcal{O}^{J / \psi}\left[{ }^{1} S_{0}^{(8)}\right]\right\rangle & =1 \times 10^{-2} \mathrm{GeV}^{3}, \\
\left\langle\mathcal{O}^{J / \psi}\left[{ }^{3} P_{0}^{(8)}\right]\right\rangle & =11.25 \times 10^{-3} \mathrm{GeV}^{5}, \\
\left\langle\mathcal{O}^{J / \psi}\left[{ }^{3} S_{1}^{(8)}\right]\right\rangle & =1.12 \times 10^{-2} \mathrm{GeV}^{3} .
\end{aligned}
$$

The LDMEs of $\psi(2 S)$ and $\chi_{c 0}$ are taken from 64 66] as

$$
\begin{aligned}
& \left\langle\mathcal{O}^{\psi^{\prime}}\left[{ }^{1} S_{0}^{(8)}\right]\right\rangle=5 \times 10^{-3} \mathrm{GeV}^{3}, \\
& \left\langle\mathcal{O}^{\psi^{\prime}}\left[{ }^{3} P_{0}^{(8)}\right]\right\rangle=3.214 \times 10^{-3} \mathrm{GeV}^{5}, \\
& \left\langle\mathcal{O}^{\psi^{\prime}}\left[{ }^{3} S_{1}^{(8)}\right]\right\rangle=2 \times 10^{-3} \mathrm{GeV}^{3},
\end{aligned}
$$

and

$$
\begin{aligned}
\left\langle\mathcal{O}^{\chi_{c 0}}\left[{ }^{3} S_{1}^{(8)}\right]\right\rangle & =2.2 \times 10^{-3} \mathrm{GeV}^{3}, \\
\left\langle\mathcal{O}^{\chi_{c 0}}\left[{ }^{3} P_{0}^{(1)}\right]\right\rangle & =\frac{3 N_{c}}{2 \pi} \times 0.075 \mathrm{GeV}^{5},
\end{aligned}
$$

with $N_{c}=3$. In accordance with Eq. (4), we can acquire the LDMEs of $\chi_{c 1}$ and $\chi_{c 2}$. In the calculation, we have considered the conventions of different theories for LDMEs of the colour singlet states.

In Tab. I. we present the cross section of the charmonium $J / \psi, \psi(2 S)$ and $\chi_{c J}(J=0,1,2)$ production in associated with the Higgs boson via $g g$ fusion from the contribution of S1 and S2 at the 14 TeV LHC within the NRQCD framework. We can find that the contribution of S2 is more than three orders of magnitudes larger than S1 in the production of charmonium associated with Higgs boson. The Fock state ${ }^{3} S_{1}^{(8)}$ plays a major role in the direct production of charmonium associated with Higgs boson via loop-induced $g g$ fusion, from which the contribution can account for more than $90 \%$ to the total direct production cross section. For the direct production of $J / \psi+H$ and $\chi_{c J}+H$ via loop-induced, the cross section can reach about $45.34 \mathrm{fb}$ and $81.82 \mathrm{fb}$ respectively, which can provide abundant and fascinating investigations of phenomenology at the LHC.

\begin{tabular}{c|c|c|c|c|c|c}
\hline \hline & Fock state & ${ }^{1} S_{0}^{(8)}$ & ${ }^{3} P_{J}^{(8)}$ & ${ }^{3} P_{J}^{(1)}$ & ${ }^{3} S_{1}^{(8)}$ & Total \\
\hline \multirow{5}{*}{$\mathrm{S} 1$} & $\sigma(p p \rightarrow g g \rightarrow J / \psi+H)$ & 0.97 & 2.64 & - & 4.84 & 8.45 \\
\cline { 2 - 7 } & $\sigma(p p \rightarrow g g \rightarrow \psi(2 S)+H)$ & 0.49 & 0.76 & - & 0.80 & 2.05 \\
\cline { 2 - 7 } & $\sigma\left(p p \rightarrow g g \rightarrow \chi_{c 0}+H\right)$ & - & - & 0.62 & 0.95 & 1.57 \\
\cline { 2 - 7 } & $\sigma\left(p p \rightarrow g g \rightarrow \chi_{c 1}+H\right)$ & - & - & 3.81 & 2.85 & 6.66 \\
\cline { 2 - 7 } & $\sigma\left(p p \rightarrow g g \rightarrow \chi_{c 2}+H\right)$ & - & - & 9.02 & 4.75 & 13.77 \\
\hline \multirow{5}{*}{$\mathrm{S} 2$} & $\sigma(p p \rightarrow g g \rightarrow J / \psi+H)$ & 0.38 & 0.71 & - & 44.25 & 45.34 \\
\cline { 2 - 7 } & $\sigma(p p \rightarrow g g \rightarrow \psi(2 S)+H)$ & 0.19 & 0.20 & - & 7.90 & 8.29 \\
\cline { 2 - 7 } & $\sigma\left(p p \rightarrow g g \rightarrow \chi_{c 0}+H\right)$ & - & - & 0.69 & 8.69 & 9.36 \\
\cline { 2 - 7 } & $\sigma\left(p p \rightarrow g g \rightarrow \chi_{c 1}+H\right)$ & - & - & 1.75 & 26.08 & 27.82 \\
\cline { 2 - 7 } & $\sigma\left(p p \rightarrow g g \rightarrow \chi_{c 2}+H\right)$ & - & - & 1.19 & 43.47 & 44.64 \\
\hline \hline
\end{tabular}

TABLE I: The cross section of charmonium $\mathcal{Q}$ production in associated with Higgs boson via $g g$ fusion from the contrition of $\mathrm{S} 1$ (in unit of $\mathrm{ab}$ ) and S2 (in unit of $\mathrm{fb}$ ) at the $14 \mathrm{TeV}$ LHC.

We present the transverse momentum $\left(p_{T}^{\mathcal{Q}}\right)$ and rapidity $\left(y_{\mathcal{Q}}\right)$ distributions of the final charmonium $\mathcal{Q}$ for the process $p p \rightarrow g g \rightarrow \mathcal{Q}+H$ via loop-induced production at the $14 \mathrm{TeV}$ LHC in Fig 3 . It can be found that, the $p_{T}$ distributions for the considered direct $\mathcal{Q}$ production all decrease rapidly with the increment of $p_{T}^{\mathcal{Q}}$. The $p_{T}$ distributions of the direct $\chi_{c 2}$ and $J / \psi$ production are almost the same in the whole $p_{T}$ region, because the Fock state ${ }^{3} S_{1}^{(8)}$ dominants the contributions and LDME values of the ${ }^{3} S_{1}^{(8)}$ for $J / \psi$ and $\chi_{c 2}$ are quite close. Due to same reason, rapidity distributions of $J / \psi$ and $\chi_{c 2}$ are also almost consistent.

In order to illustrate the transverse momentum $p_{T}$ and rapidity $y$ distributions from each Fock state, we take direct $J / \psi$ production in associated with Higgs boson as an example and present the contribution from Fock states ${ }^{1} S_{0}^{(8)}$, ${ }^{3} P_{J}^{(8)}$ and ${ }^{3} S_{1}^{(8)}$ for $J / \psi$ in Fig. 4, separately. The production channel of ${ }^{3} S_{1}^{(1)}+H$ is forbidden due to chargeconjugation invariance. We can see that the contribution from each Fock state decreases rapidly with the increment 

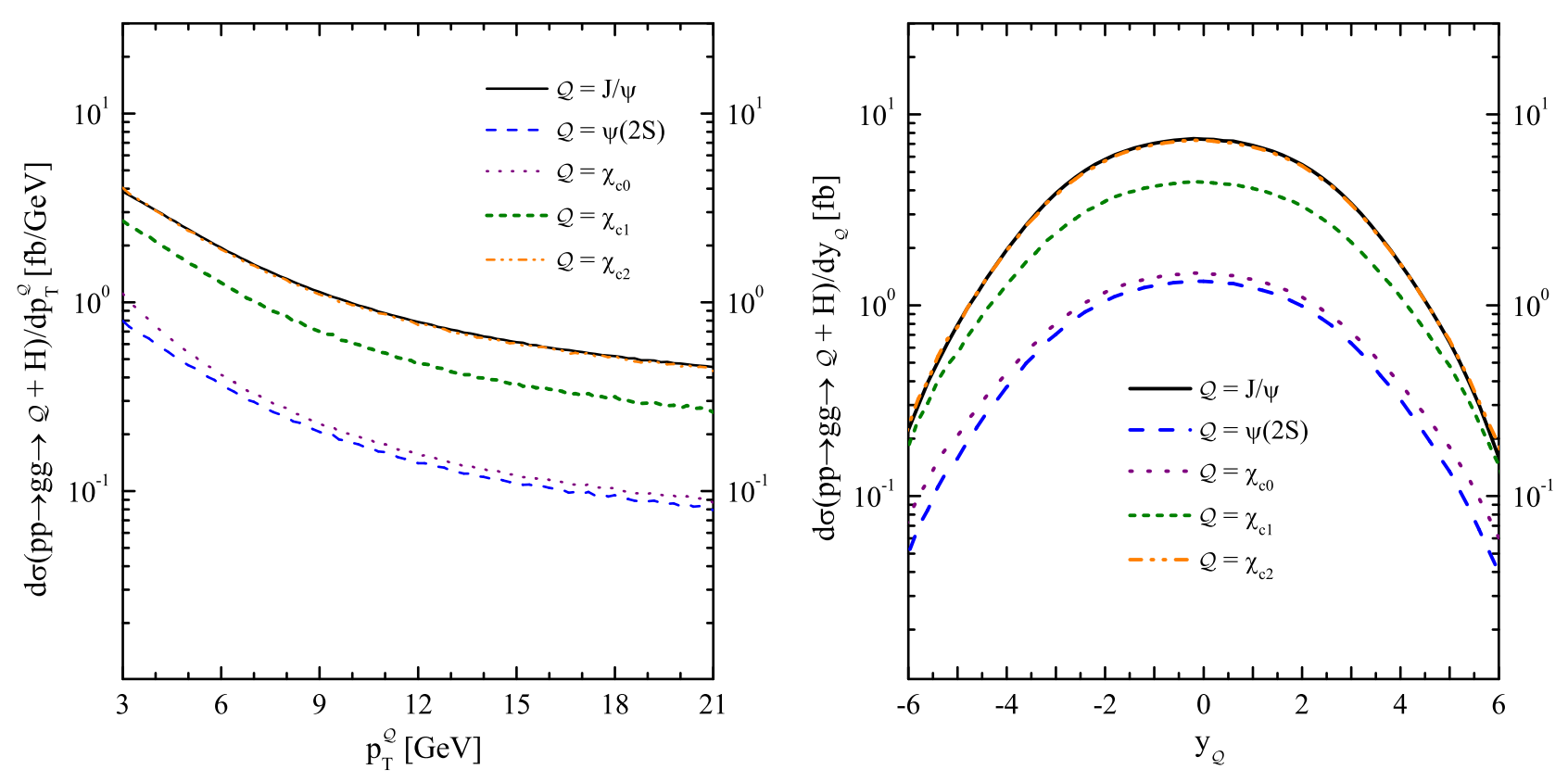

FIG. 3: The distributions of $p_{T}^{\mathcal{Q}}$ (left) and $y_{\mathcal{Q}}$ (right) for the process $p p \rightarrow g g \rightarrow \mathcal{Q}+H$ via loop-induced at the 14 TeV LHC. The black solid, blue dashed, purple dotted, olive short dashed and orange dashed-dotted-dotted lines denote the final charmonium $\mathcal{Q}$ are $J / \psi, \psi(2 S), \chi_{c 0}, \chi_{c 1}$ and $\chi_{c 2}$, respectively.

of $p_{T}^{J / \psi}$, but ${ }^{3} S_{1}^{(8)}$ decreases slower than ${ }^{1} S_{0}^{(8)}$ and ${ }^{3} P_{J}^{(8)}$. In the range of $3 \mathrm{GeV} \leq p_{T}^{J / \psi} \leq 15 \mathrm{GeV}$, the differential cross section of $J / \psi$ associated with Higgs boson production lies in the range of $[0.62,3.85] \mathrm{fb} / \mathrm{GeV}$. From Fig. [4, we can find that the contribution from ${ }^{3} S_{1}^{(8)}$ Fock state is always dominant, especially at large $p_{T}$ the contribution due to the other Fock states can be negligible. It implies that this process will help to extract the LDMEs of ${ }^{3} S_{1}^{(8)}$ Fock state if it is detected in future experiments.

We respectively present the total cross section for the prompt $J / \psi$ associated with Higgs boson via $g g$ fusion from the contribution of S1 and S2 at the $14 \mathrm{TeV}$ LHC in Tab. II The contributions due to direct and indirect production are also shown respectively. From Tab. II we can see that for S1, even considering the direct and indirect contributions, the cross section of prompt $J / \psi+H$ production is still tiny compared to S2. As for the contribution of $\mathrm{S} 2$, we can find that direct production is dominant, and can reach about $66 \%$ of the prompt production. Indirect production due to excited charmonium decays is not negligible, whose rate can account for about $34 \%$. In indirect production, the contributions of $\psi(2 S)+H, \chi_{c 1}+H$ and $\chi_{c 2}+H$ are sizable, which are about $22 \%, 41 \%$ and $36 \%$ of the indirect contribution, respectively. The indirect contribution from $\chi_{c 0}+H$ is less than $0.2 \%$ of the prompt production, because the decay branching ratio $\operatorname{Br}\left(\chi_{c 0} \rightarrow J / \psi+\gamma\right)$ is tiny. Considering the contribution from direct and indirect productions, the total cross section of prompt $J / \psi+H$ production can reach $68.58 \mathrm{fb}$.

We further consider the final Higgs boson decaying into $b \bar{b}$. For the $b$-jets, applying the cuts of $p_{T}^{b}>20 \mathrm{GeV},\left|\eta_{b}\right|<$ 2.5 , and considering the branching ratio $\operatorname{Br}(H \rightarrow b \bar{b})=58.14 \%[67]$ and b-tagging efficiency $\epsilon_{b}=77 \%$ [68], we can get the cross section from S2 as 11.12 (5.7) fb for the direct (indirect) production of $J / \psi$ associated with Higgs boson via $g g$ fusion. Therefore, the cross section of prompt $J / \psi+H$ with Higgs boson decaying into $b \bar{b}$ can reach about $18 \mathrm{fb}$ even after considering the kinematical constraints on the Higgs in experiments. This implies that this process may be detected in future experiments, which will help us to further understand the colour-octet mechanism.

\begin{tabular}{c|c|c|c|c|c|c|c}
\hline \hline Source & From $\psi(2 S)+H$ & From $\chi_{c 0}+H$ & From $\chi_{c 1}+H$ & From $\chi_{c 2}+H$ & Indirect & Direct & Prompt \\
\hline$\sigma(S 1)$ & 1.26 & 0.02 & 2.28 & 2.62 & 6.18 & 8.45 & 14.63 \\
\hline$\sigma(S 2)$ & 5.09 & 0.13 & 9.54 & 8.48 & 23.24 & 45.34 & 68.58 \\
\hline \hline
\end{tabular}

TABLE II: The cross sections for the prompt $J / \psi$ associated with Higgs boson via $g g$ fusion including the contribution of S1 (in unit of $\mathrm{ab}$ ) and S2 (in unit of $\mathrm{fb}$ ) from direct and indirect production at the $14 \mathrm{TeV}$ LHC, respectively.

The distributions of $p_{T}^{J / \psi}$ (left) and $y_{J / \psi}$ (right) for direct and indirect productions of the $J / \psi$ associated with 

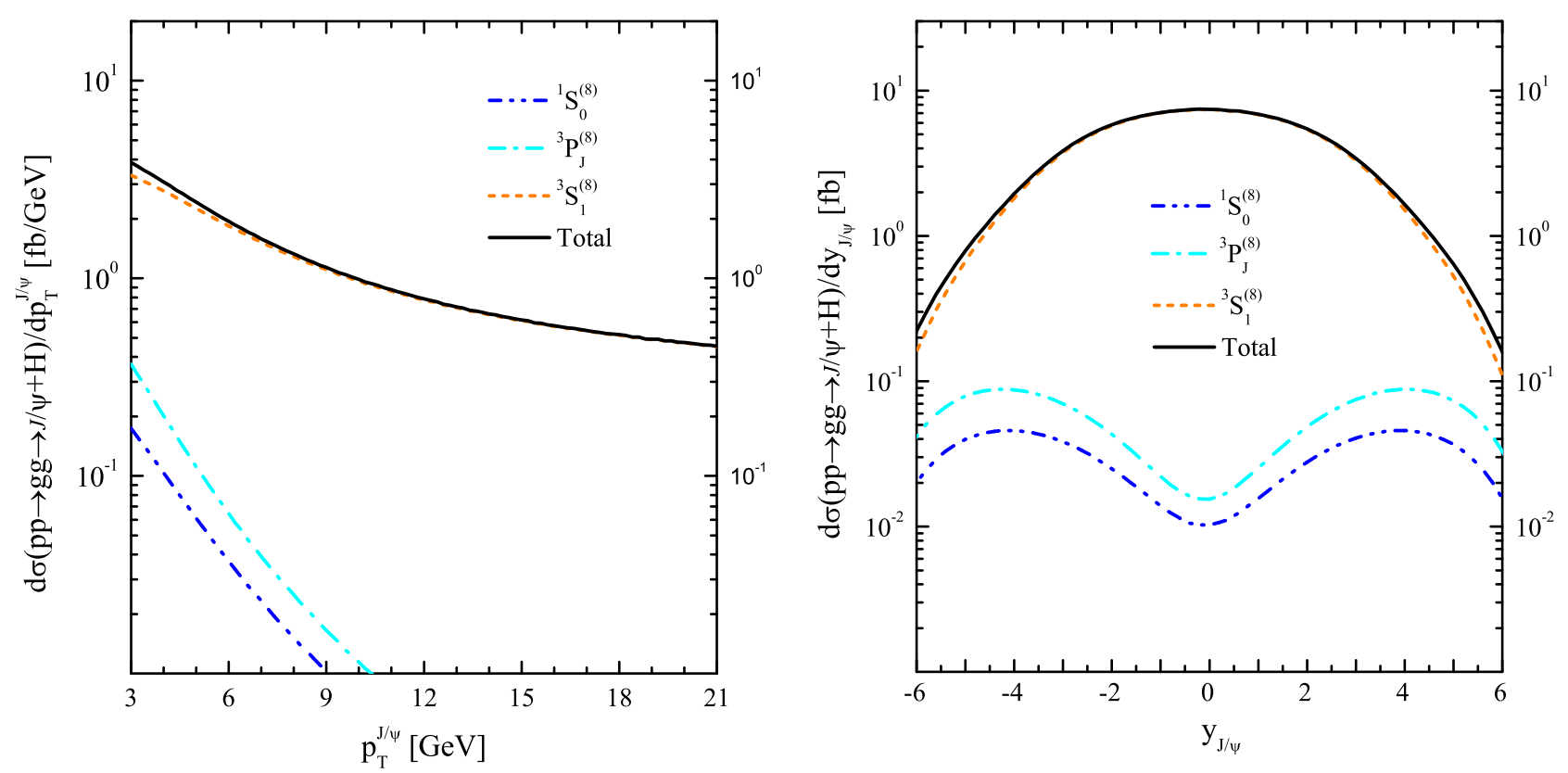

FIG. 4: The distributions of $p_{T}^{J / \psi}$ (left) and $y_{J / \psi}$ (right) for direct production of the $J / \psi$ associated with Higgs boson via loop-induced at the $14 \mathrm{TeV}$ LHC. The blue dashed-dotted-dotted, cyan dashed-dotted, orange dashed and black solid lines are for ${ }^{1} S_{0}^{(8)},{ }^{3} P_{J}^{(8)},{ }^{3} S_{1}^{(8)}$ and total, respectively.
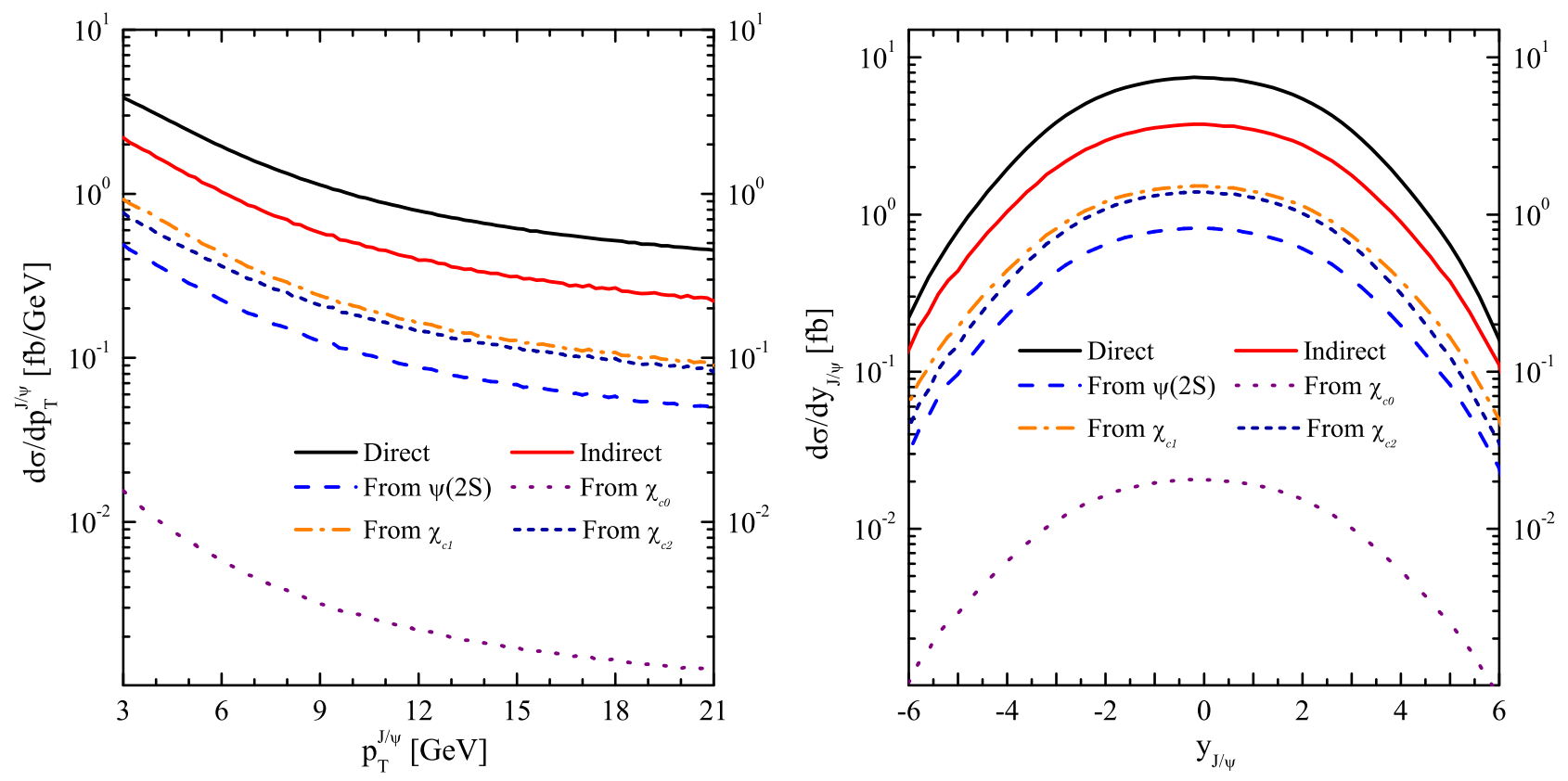

FIG. 5: The distributions of $p_{T}^{J / \psi}$ (left) and $y_{J / \psi}$ (right) for direct and indirect contribution of the $J / \psi$ associated with Higgs boson via loop-induced at the $14 \mathrm{TeV}$ LHC. The black solid, red solid, blue dashed, purple dotted, orange dashed-dotted and royal short dashed lines are contributions of from direct, indirect, $\psi(2 S), \chi_{c 0}, \chi_{c 1}$ and $\chi_{c 2}$.

Higgs boson via loop-induced at the $14 \mathrm{TeV}$ LHC are shown in Fig 5 , It can be seen that the behavior of the $p_{T}^{J / \psi}$ and $y_{J / \psi}$ distribution for the direct and indirect productions are similar. The curves of the $J / \psi+H$ direct production are always at the top in the whole plotted region of $p_{T}^{J / \psi}$ and $y_{J / \psi}$. The indirect contributions from $\psi(2 S), \chi_{c 1}$ and $\chi_{c 2}$ are considerable, while from $\chi_{c 0}$ is much less than from other charmonia because of the small decay branching 
ratio. For the $y_{J / \psi}$ distributions, the convex curves of the direct and indirect contributions reach their peaks when $y_{J / \psi}=0$.

\begin{tabular}{c|c|c|c|c}
\hline \hline & Set 1 & Set 2 & Set 3 & Default set \\
\hline$<\mathcal{O}^{J / \psi}\left[{ }^{1} S_{0}^{[8]}\right]>\left(\mathrm{GeV}^{3}\right)$ & $4.35 \times 10^{-2}$ & $5.45 \times 10^{-2}$ & $0.78 \times 10^{-2}$ & $1 \times 10^{-2}$ \\
\hline$<\mathcal{O}^{J / \psi}\left[{ }^{3} P_{0}^{[8]}\right]>\left(\mathrm{GeV}^{5}\right)$ & $2.879 \times 10^{-2}$ & $3.50 \times 10^{-2}$ & $4.3515 \times 10^{-2}$ & $1.125 \times 10^{-2}$ \\
\hline$<\mathcal{O}^{J / \psi}\left[{ }^{3} S_{1}^{[8]}\right]>\left(\mathrm{GeV}^{3}\right)$ & $4.4 \times 10^{-3}$ & $1.4 \times 10^{-2}$ & $1.057 \times 10^{-2}$ & $1.12 \times 10^{-2}$ \\
\hline$\sigma(p p \rightarrow g g \rightarrow J / \psi+H)(\mathrm{fb})$ & 20.85 & 59.59 & 44.81 & 45.34 \\
\hline \hline
\end{tabular}

TABLE III: The cross sections (fb) of the direct $J / \psi$ associated with Higgs boson production via $g g$ fusion from the contribution of S2 at the $14 \mathrm{TeV}$ LHC with four different sets of LDMEs, in which Set 1 and Set 2 are extracted by fitting the $p_{T}$ distributions from CDF using PDFs for MRST98LO[69] and MRS(R2) [70], respectively. And Set 3 is extracted from the hadroproduction of $\eta_{c}$ at the LHC[71].

As we know, in the study of heavy quarkonium production and decay processes, LDMEs is considered universal. The LDMEs of colour octet are extracted from experiments, which lead to the uncertainty. In order to study the uncertainty of the results caused by the LDMEs, we present the cross sections of the direct $J / \psi$ associated with Higgs boson production via $g g$ fusion from the contribution of $\mathrm{S} 2$ at the $14 \mathrm{TeV}$ LHC with other three different sets of LDMEs in Tab. III] Set 1 and Set 2 are extracted by fitting the $p_{T}$ distributions from CDF using PDFs for MRST98LO 69] and MRS(R2) [70], respectively; Set 3 is extracted from the hadroproduction of $\eta_{c}$ at the LHC[71]. From Tab. III, we can see that the cross section of $J / \psi+H$ varies between $46 \%-131 \%$ compared with the result with the default set of LDMEs. Moreover, we take the production channel of $J / \psi\left({ }^{3} S_{1}^{(8)}\right)$ as an example to study the uncertainty due to PDFs. In Tab. IV] we respectively show the cross sections of $J / \psi\left({ }^{3} S_{1}^{(8)}\right)+H$ via $g g$ fusion from the contribution of S2 at the $14 \mathrm{TeV}$ LHC with other three different PDFs. From Tab. IV we can find that the results are slightly affected by the different PDFs. In addition to the LDMEs and PDFs mentioned above, the numerical uncertainty in our calculation is also affected by the factorization scale and quark mass.

\begin{tabular}{c|c|c|c|c}
\hline \hline PDFs & MSHT20lo_as130[72] & NNPDF30lo_as_0130[73] & NNPDF31_lo_pch_as_0130[74] & Default \\
\hline$\sigma\left(p p \rightarrow g g \rightarrow J / \psi\left({ }^{3} S_{1}^{(8)}\right)+H\right)(\mathrm{fb})$ & 45.01 & 43.50 & 42.65 & 44.25 \\
\hline \hline
\end{tabular}

TABLE IV: The cross sections of the $J / \psi\left({ }^{3} S_{1}^{(8)}\right)+H$ production channel via $g g$ fusion from the contribution of S2 at the 14 $\mathrm{TeV}$ LHC with four different PDFs, respectively.

\section{SUMMARY AND DISCUSSION}

In this paper, we revisit the production of prompt $J / \psi$ associated with Higgs boson via $g g$ fusion include the contribution of S1 and S2 at the $14 \mathrm{TeV}$ LHC within the framework of NRQCD. We consider the direct contribution from $J / \psi+H$ and indirect contribution from $\psi(2 S)+H$ and $\chi_{c J}+H$. From our calculations, we find that the contribution of S2 is more than three orders of magnitudes larger than S1 for the production of charmonium associated with Higgs boson. Not like the contribution of S1, which is to be far too rare to be observable, the contribution of $\mathrm{S} 2$ has great potential to be detected. We not only give the total cross section of prompt $J / \psi+H$, but also present the distribution of transverse momentum and rapidity for the final $J / \psi$ in the contribution of S2. We find that the total cross section of prompt $J / \psi+H$ can reach about $70 \mathrm{fb}$ and the ${ }^{3} S_{1}^{(8)}$ Fock state gives the dominant contribution in all production channels. In the process of prompt $J / \psi+H$ production via loop-induced, the direct contribution is a majority, while the total indirect contribution is also considerable, accounting for $34 \%$ of the prompt $J / \psi+H$. The productions of $\psi(2 S)+H, \chi_{c 1}+H$ and $\chi_{c 2}+H$ provide $22 \%, 41 \%$ and $36 \%$ of the total indirect contribution, respectively. Since the decay channel of $J / \psi$ is clean, it is possible to detect the production of $J / \psi$ associated with the Higgs boson at the LHC in the future, which will help us to further understand the mechanism of colour-octet, as well as help us to further investigate the coupling of the Higgs boson and fermion. 


\section{Acknowledgments}

This work was supported in part by the National Natural Science Foundation of China (Grants No. 11805001, No. 11935001, No.11875070).

[1] B. A. Kniehl, C. P. Palisoc and L. Zwirner, Phys. Rev. D 66, 114002 (2002) doi:10.1103/PhysRevD.66.114002 arXiv:hep-ph/0208104 [hep-ph]].

[2] J. J. Aubert et al. [E598], Phys. Rev. Lett. 33, 1404-1406 (1974) doi:10.1103/PhysRevLett.33.1404

[3] J. E. Augustin et al. [SLAC-SP-017], Phys. Rev. Lett. 33, 1406-1408 (1974) doi:10.1103/PhysRevLett.33.1406

[4] B. Aubert et al. [BaBar], Phys. Rev. Lett. 87, 162002 (2001) doi:10.1103/PhysRevLett.87.162002 arXiv:hep-ex/0106044 [hep-ex]].

[5] K. Abe et al. [Belle], Phys. Rev. Lett. 88, 052001 (2002) doi:10.1103/PhysRevLett.88.052001 arXiv:hep-ex/0110012 [hepex]].

[6] S. Aid et al. [H1], Nucl. Phys. B 472, 3-31 (1996) doi:10.1016/0550-3213(96)00274-X arXiv:hep-ex/9603005 [hep-ex]].

[7] S. Aid et al. [H1], Nucl. Phys. B 468, 3-36 (1996) [erratum: Nucl. Phys. B 548, 639-639 (1999)] doi:10.1016/05503213(96)00192-7 arXiv:hep-ex/9602007 [hep-ex]].

[8] C. Adloff et al. [H1], Phys. Lett. B 483, 23-35 (2000) doi:10.1016/S0370-2693(00)00530-X arXiv:hep-ex/0003020 [hep-ex]].

[9] A. Aktas et al. [H1], Eur. Phys. J. C 46, 585-603 (2006) doi:10.1140/epjc/s2006-02519-5 arXiv:hep-ex/0510016 [hep-ex]].

[10] C. Alexa et al. [H1], Eur. Phys. J. C 73, no.6, 2466 (2013) doi:10.1140/epjc/s10052-013-2466-y [arXiv:1304.5162 [hep-ex]].

[11] S. Chekanov et al. [ZEUS], Eur. Phys. J. C 24, 345-360 (2002) doi:10.1007/s10052-002-0953-7 arXiv:hep-ex/0201043 [hep-ex]].

[12] V. Khachatryan et al. [CMS], Phys. Rev. D 83, 112004 (2011) doi:10.1103/PhysRevD.83.112004 arXiv:1012.5545 [hep-ex]].

[13] R. Aaij et al. [LHCb], Eur. Phys. J. C 74, no.5, 2872 (2014) doi:10.1140/epjc/s10052-014-2872-9 arXiv:1403.1339 [hep-ex]].

[14] T. Aaltonen et al. [CDF], Phys. Rev. Lett. 108, 151802 (2012) doi:10.1103/PhysRevLett.108.151802 arXiv:1112.1591 [hep-ex]].

[15] A. Abulencia et al. [CDF], Phys. Rev. Lett. 99, 132001 (2007) doi:10.1103/PhysRevLett.99.132001 arXiv:0704.0638 [hepex]].

[16] R. Aaij et al. [LHCb], Eur. Phys. J. C 73, no.11, 2631 (2013) doi:10.1140/epjc/s10052-013-2631-3 arXiv:1307.6379 [hep$\mathrm{ex}]$.

[17] K. Abe et al. [Belle], Phys. Rev. D 70, 071102 (2004) doi:10.1103/PhysRevD.70.071102 arXiv:hep-ex/0407009 [hep-ex]].

[18] R. Aaij et al. [LHCb], Phys. Lett. B 707, 52-59 (2012) doi:10.1016/j.physletb.2011.12.015 [arXiv:1109.0963 [hep-ex]].

[19] V. Khachatryan et al. [CMS], JHEP 09, 094 (2014) doi:10.1007/JHEP09(2014)094 arXiv:1406.0484] [hep-ex]].

[20] V. M. Abazov et al. [D0], Phys. Rev. D 90, no.11, 111101 (2014) doi:10.1103/PhysRevD.90.111101 arXiv:1406.2380 [hep-ex]].

[21] B. Aubert et al. [BaBar], Phys. Rev. D 72, 031101 (2005) doi:10.1103/PhysRevD.72.031101 arXiv:hep-ex/0506062 [hep$\mathrm{ex}]$.

[22] K. Abe et al. [Belle], Phys. Rev. Lett. 89, 142001 (2002) doi:10.1103/PhysRevLett.89.142001 arXiv:hep-ex/0205104 [hep$\mathrm{ex}]$.

[23] V. D. Barger, W. Y. Keung and R. J. N. Phillips, Phys. Lett. B 91, 253-258 (1980) doi:10.1016/0370-2693(80)90444-X

[24] V. D. Barger, W. Y. Keung and R. J. N. Phillips, Z. Phys. C 6, 169 (1980) doi:10.1007/BF01588844

[25] M. Ablikim et al. [BESIII], Phys. Rev. Lett. 125, no.5, 052004 (2020) doi:10.1103/PhysRevLett.125.052004 arXiv:2004.07701 [hep-ex]].

[26] E. L. Berger and D. L. Jones, Phys. Rev. D 23, 1521-1530 (1981) doi:10.1103/PhysRevD.23.1521

[27] G. T. Bodwin, E. Braaten and G. P. Lepage, Phys. Rev. D 51, 1125-1171 (1995) [erratum: Phys. Rev. D 55, 5853 (1997)] doi:10.1103/PhysRevD.55.5853 arXiv:hep-ph/9407339 [hep-ph]].

[28] E. Braaten and S. Fleming, Phys. Rev. Lett. 74, 3327-3330 (1995) doi:10.1103/PhysRevLett.74.3327 arXiv:hep-ph/9411365 [hep-ph]].

[29] P. L. Cho and A. K. Leibovich, Phys. Rev. D 53, 150-162 (1996) doi:10.1103/PhysRevD.53.150 arXiv:hep-ph/9505329 [hep-ph]].

[30] J. P. Lansberg, Int. J. Mod. Phys. A 21, 3857-3916 (2006) doi:10.1142/S0217751X06033180 arXiv:hep-ph/0602091 [hep$\mathrm{ph}]$.

[31] J. P. Lansberg, Phys. Rept. 889, 1-106 (2020) doi:10.1016/j.physrep.2020.08.007 arXiv:1903.09185 [hep-ph]].

[32] A. Usachov, arXiv:1910.08796 [hep-ex]].

[33] L. P. Sun, H. Han and K. T. Chao, Phys. Rev. D 94, no.7, 074033 (2016) doi:10.1103/PhysRevD.94.074033 arXiv:1404.4042 [hep-ph]].

[34] J. P. Lansberg and H. S. Shao, Phys. Rev. Lett. 111, 122001 (2013) doi:10.1103/PhysRevLett.111.122001 arXiv:1308.0474 [hep-ph]].

[35] Y. Q. Ma, K. Wang and K. T. Chao, Phys. Rev. Lett. 106, 042002 (2011) doi:10.1103/PhysRevLett.106.042002 arXiv:1009.3655 [hep-ph]].

[36] Y. J. Zhang, Y. Q. Ma, K. Wang and K. T. Chao, Phys. Rev. D 81, 034015 (2010) doi:10.1103/PhysRevD.81.034015 
arXiv:0911.2166 [hep-ph]].

[37] D. P. Roy and K. Sridhar, Phys. Lett. B 341, 413-418 (1995) doi:10.1016/0370-2693(94)01321-3 arXiv:hep-ph/9407390 [hep-ph]].

[38] C. S. Kim, J. Lee and H. S. Song, Phys. Rev. D 55, 5429-5436 (1997) doi:10.1103/PhysRevD.55.5429 arXiv:hep-ph/9610294 [hep-ph]].

[39] E. Braaten, J. Lee and S. Fleming, Phys. Rev. D 60, 091501 (1999) doi:10.1103/PhysRevD.60.091501 arXiv:hep-ph/9812505 [hep-ph]].

[40] R. Li and J. X. Wang, Phys. Lett. B 672, 51-55 (2009) doi:10.1016/j.physletb.2008.12.050 arXiv:0811.0963 [hep-ph]].

[41] G. Li, M. Song, R. Y. Zhang and W. G. Ma, Phys. Rev. D 83, 014001 (2011) doi:10.1103/PhysRevD.83.014001 arXiv:1012.3798 [hep-ph]].

[42] M. Song, W. G. Ma, G. Li, R. Y. Zhang and L. Guo, JHEP 02, 071 (2011) [erratum: JHEP 12, 010 (2012)] doi:10.1007/JHEP02(2011)071 arXiv:1102.0398 [hep-ph]].

[43] G. Li, W. G. Ma, M. Song, R. Y. Zhang and J. Y. Guo, JHEP 01, 034 (2013) doi:10.1007/JHEP01(2013)034 arXiv:1212.2417 [hep-ph]].

[44] M. Song, G. Li, W. G. Ma, R. Y. Zhang, L. Guo and J. Y. Guo, Chin. Phys. Lett. 30, 091201 (2013) doi:10.1088/0256307X/30/9/091201 arXiv:1304.4670 [hep-ph]].

[45] J. P. Lansberg and H. S. Shao, JHEP 10, 153 (2016) doi:10.1007/JHEP10(2016)153 arXiv:1608.03198] [hep-ph]].

[46] J. P. Lansberg, H. S. Shao and N. Yamanaka, Phys. Lett. B 781, 485-491 (2018) doi:10.1016/j.physletb.2018.04.020 arXiv:1707.04350 [hep-ph]].

[47] G. Aad et al. [ATLAS], JHEP 04, 172 (2014) doi:10.1007/JHEP04(2014)172 arXiv:1401.2831 [hep-ex]].

[48] G. Aad et al. [ATLAS], Eur. Phys. J. C 75, no.5, 229 (2015) doi:10.1140/epjc/s10052-015-3406-9 arXiv:1412.6428 [hep-ex]].

[49] M. Aaboud et al. [ATLAS], JHEP 01, 095 (2020) doi:10.1007/JHEP01(2020)095 arXiv:1909.13626 [hep-ex]].

[50] J. P. Lansberg and C. Lorce, Phys. Lett. B 726, 218-222 (2013) [erratum: Phys. Lett. B 738, 529-529 (2014)] doi:10.1016/j.physletb.2013.07.059 [arXiv:1303.5327 [hep-ph]].

[51] B. A. Kniehl, C. P. Palisoc and L. Zwirner, Phys. Rev. D 69, 115005 (2004) doi:10.1103/PhysRevD.69.115005 arXiv:hep-ph/0404039 [hep-ph]].

[52] M. Tanabashi et al. [Particle Data Group], Phys. Rev. D 98, no.3, 030001 (2018) doi:10.1103/PhysRevD.98.030001

[53] A. Petrelli, M. Cacciari, M. Greco, F. Maltoni and M. L. Mangano, Nucl. Phys. B 514, 245-309 (1998) doi:10.1016/S05503213(97)00801-8 [arXiv:hep-ph/9707223 [hep-ph]].

[54] T. Hahn, Comput. Phys. Commun. 140, 418-431 (2001) doi:10.1016/S0010-4655(01)00290-9 arXiv:hep-ph/0012260 [hep$\mathrm{ph}]]$.

[55] V. Shtabovenko, R. Mertig and F. Orellana, Comput. Phys. Commun. 207, 432-444 (2016) doi:10.1016/j.cpc.2016.06.008 arXiv:1601.01167 [hep-ph]].

[56] F. Feng, Comput. Phys. Commun. 183, 2158-2164 (2012) doi:10.1016/j.cpc.2012.03.025 arXiv:1204.2314 [hep-ph]].

[57] F. Feng, J. Phys. Conf. Ser. 523, 012041 (2014) doi:10.1088/1742-6596/523/1/012041 arXiv:1307.5587 [hep-ph]].

[58] A. V. Smirnov, JHEP 10, 107 (2008) doi:10.1088/1126-6708/2008/10/107 arXiv:0807.3243 [hep-ph]].

[59] J. Fleischer, F. Jegerlehner and O. V. Tarasov, Nucl. Phys. B 566, 423-440 (2000) doi:10.1016/S0550-3213(99)00678-1 arXiv:hep-ph/9907327 [hep-ph]].

[60] T. Hahn and M. Perez-Victoria, Comput. Phys. Commun. 118, 153-165 (1999) doi:10.1016/S0010-4655(98)00173-8 arXiv:hep-ph/9807565 [hep-ph]].

[61] J. Pumplin, D. R. Stump, J. Huston, H. L. Lai, P. M. Nadolsky and W. K. Tung, JHEP 07, 012 (2002) doi:10.1088/11266708/2002/07/012 arXiv:hep-ph/0201195 [hep-ph]].

[62] S. Fleming and T. Mehen, Phys. Rev. D 57, 1846-1857 (1998) doi:10.1103/PhysRevD.57.1846 arXiv:hep-ph/9707365 [hep-ph]].

[63] B. A. Kniehl and L. Zwirner, Nucl. Phys. B 621, 337-358 (2002) doi:10.1016/S0550-3213(01)00564-8 arXiv:hep-ph/0112199 [hep-ph]].

[64] B. A. Kniehl and C. P. Palisoc, Eur. Phys. J. C 48, 451-456 (2006) doi:10.1140/epjc/s10052-006-0044-2 arXiv:hep-ph/0608245 [hep-ph]].

[65] E. J. Eichten and C. Quigg, Phys. Rev. D 52, 1726-1728 (1995) doi:10.1103/PhysRevD.52.1726 arXiv:hep-ph/9503356 [hep-ph]].

[66] Y. Q. Ma, K. Wang and K. T. Chao, Phys. Rev. D 83, 111503 (2011) doi:10.1103/PhysRevD.83.111503 arXiv:1002.3987 [hep-ph]].

[67] S. Dawson, C. Englert and T. Plehn, Phys. Rept. 816, 1-85 (2019) doi:10.1016/j.physrep.2019.05.001 arXiv:1808.01324 [hep-ph]].

[68] G. Li, X. A. Pan, M. Song and Y. Zhang, Phys. Rev. D 100, no.7, 074019 (2019) doi:10.1103/PhysRevD.100.074019 arXiv:1904.10198 [hep-ph]].

[69] E. Braaten, B. A. Kniehl and J. Lee, Phys. Rev. D 62, 094005 (2000) doi:10.1103/PhysRevD.62.094005 arXiv:hep-ph/9911436 [hep-ph]].

[70] M. Beneke and M. Krämer, Phys. Rev. D 55, 5269-5272 (1997) doi:10.1103/PhysRevD.55.R5269 arXiv:hep-ph/9611218 [hep-ph]].

[71] Z. Sun, X. G. Wu and H. F. Zhang, Phys. Rev. D 92, no.7, 074021 (2015) doi:10.1103/PhysRevD.92.074021 arXiv:1507.08190 [hep-ph]].

[72] S. Bailey, T. Cridge, L. A. Harland-Lang, A. D. Martin and R. S. Thorne, Eur. Phys. J. C 81, no.4, 341 (2021) doi:10.1140/epjc/s10052-021-09057-0 [arXiv:2012.04684 [hep-ph]]. 
[73] R. D. Ball et al. [NNPDF], JHEP 04, 040 (2015) doi:10.1007/JHEP04(2015)040 arXiv:1410.8849 [hep-ph]].

[74] R. D. Ball et al. [NNPDF], Eur. Phys. J. C 77, no.10, 663 (2017) doi:10.1140/epjc/s10052-017-5199-5 arXiv:1706.00428 [hep-ph]]. 